\title{
Costruiamo un carretto
}

\section{Let's build a trolley cart}

\author{
Laura Battaini \\ Scuola dell'infanzia - Pregassona, Svizzera
}

Sunto / Il presente contributo ha lo scopo di descrivere un percorso didattico rivolto sia alla scuola dell'infanzia, sia alla scuola elementare. Il progetto, che ha visto coinvolte una sezione di scuola dell'infanzia e una classe di quinta elementare di Pregassona, verte sulla costruzione di un carretto per trasportare alcuni materiali dalla sede della scuola dell'infanzia a quella della scuola elementare. Grazie alla sperimentazione e alla collaborazione, gli allievi hanno affrontato molti aspetti, matematici e tecnici, per arrivare alla costruzione di un carretto in legno adatto allo scopo.

Parole chiave: laboratorio; collaborazione; situazione - problema; curriculum verticale.

\begin{abstract}
This contribution aims to present an educational experience designed for both kindergarten and primary school. The project, conducted in a kindergarten section and in a fifth grade classroom in Pregassona, dealt with the construction of a cart to transport materials from the kindergarten to the primary school. Thanks to experimentation and collaboration, the students have faced many mathematical and technical aspects in order to construct a wooden cart suitable for the purpose.
\end{abstract}

Keywords: laboratory; collaboration; realistic situation; vertical curriculum.

In questo articolo presentiamo un percorso svolto in collaborazione tra una sezione di scuola dell'infanzia e una classe di quinta elementare del Canton Ticino con lo scopo di permettere agli allievi di avere un progetto comune, il più concreto possibile, nel quale mettere in gioco le proprie conoscenze e abilità, lavorando sulla collaborazione tra cicli diversi e sul riconoscimento delle proprie e altrui capacità. Le finalità di un percorso condiviso tra allievi di età diversa riguardano in primo luogo la valenza a livello di scambio e conoscenza tra ordini scolastici differenti; tale conoscenza reciproca si dimostra utile per gli alunni ma anche per i docenti, che hanno l'occasione di lavorare nell'ottica di un'armonizzazione e di una verticalità dell'esperienza educativa. D'altra parte, sfruttando la grande differenza d'età, questo tipo di progetti offre la possibilità di far accrescere competenze collaborative tra gli alunni. Per i bambini di scuola dell'infanzia, la scuola elementare è un mondo molto vicino ma al tempo stesso sconosciuto. Per gli allievi dell'ultimo anno in particolare, che sono consapevoli di doverla frequentare in un immediato futuro, ci sono dunque aspettative e curiosità, ma anche paure e dubbi. Numerose esperienze hanno mostrato come la maggiore conoscenza del nuovo contesto porti i bambini a essere più sereni al momento di questo delicato passaggio. Anche per questo motivo si è cercato, negli ultimi anni, di attivare frequenti scambi e collaborazioni fra i due livelli. In questo modo, si cerca di garantire agli allievi di tutte le età della scuola dell'infanzia la possibilità di conoscere meglio la scuola elementare e capire le principali differenze 
e similitudini con il loro settore di appartenenza.

Va anche considerato che i progetti che prevedono uno scambio tra i due settori scolastici sono sempre apprezzati dagli allievi, sia dai più piccoli, sia dai più grandi. Oltre all'aspetto, già evidenziato, di maggiore conoscenza e consapevolezza rispetto a un ambiente nel quale saranno inseriti in seguito, per i più piccoli c'è anche il piacere di mettersi a confronto con ragazzi più grandi, contribuendo con il proprio vissuto e le proprie idee alla risoluzione di una problematica comune, e sentendo riconosciute e valorizzate le proprie capacità. Per gli alunni più grandi invece, oltre al valore affettivo di ritornare a un ambiente legato al proprio passato educativo ed esperienziale, vi è una valorizzazione delle proprie conoscenze e competenze, che saranno maggiori e più articolate rispetto a quelle dei piccoli. Tali scambi, che si verificano quindi non solo sul piano affettivo ma anche sul piano cognitivo, sono resi possibili dalla verticalità del curricolo promossa dalle indicazioni previste dal Piano di studio della scuola dell'obbligo ticinese (DECS, 2015).

Abbiamo potuto notare come il lavoro tra bambini di età diverse sia molto efficace per sviluppare le capacità di collaborazione, in particolare nei ragazzi che mostrano difficoltà in questa competenza trasversale. Se si collabora con un pari si può faticare a immedesimarsi nelle sue difficoltà o a capire che ciò che si sta dicendo non è chiaro per l'interlocutore. Quando invece si lavora con qualcuno di distante dalla propria situazione, come è per esempio un bambino di scuola dell'infanzia per un ragazzo di quinta elementare, si parte già dal presupposto che sia a un livello radicalmente diverso dal proprio; si dovranno quindi trovare altre parole, altri modi, altri mezzi per farsi capire; ci si dovrà mettere nei panni dell'altro, capire il suo livello, le sue difficoltà e adeguarsi a esse (Arrigo, Maurizi \& Minazzi, 2005).

Chiaramente, questi passaggi sono gli stessi che bisognerebbe fare per collaborare con un pari: partire dal suo livello e cercare delle modalità a lui congeniali. Tuttavia, I'esasperazione di questa differenza, resa esplicita dalla lontananza d'età, ha permesso che gli allievi si accorgessero di questi aspetti e provassero (non sempre con successo) a fare un passo verso l'altro. Per chi ha difficoltà da questo punto di vista, il lavoro con allievi più piccoli è un modo per cercare di migliorare le proprie capacità, nella speranza di poter poi applicare le competenze acquisite anche con i pari e rendere la collaborazione più proficua ed efficace (Cacciamani, 2008).

Per quanto riguarda i concetti matematici sui quali era incentrato il percorso, si è potuto notare come l'esigenza di dover spiegare, semplificare, andare alla base del concetto, abbia permesso a molti allievi di approfondire meglio ciò che avevano trattato, favorendo una maggiore comprensione.

Nel progetto che abbiamo proposto è stata molto interessante e centrale la valorizzazione dei ragazzi di quinta; essi hanno dovuto in primo luogo mettere le proprie conoscenze e competenze al servizio dei bambini di scuola dell'infanzia adattando il proprio linguaggio al livello cognitivo di chi avevano di fronte; d'altra parte, agli alunni delle elementari era richiesto di affiancare i bambini più piccoli senza che questo significasse sostituirsi a loro nella risoluzione della situazione - problema: ai ragazzi di quinta elementare è stato quindi chiesto di non prendere decisioni ma guidare, consigliare e descrivere le diverse scelte attuabili; questa dimensione è stata molto complessa per alcuni allievi che hanno faticato ad accettare il fatto che il risultato di un processo potesse essere diverso da ciò che volevano o avrebbero potuto ottenere in modo autonomo. Al tempo stesso, però, è stato dato molto valore alle conoscenze degli allievi di scuola dell'infanzia, che durante il percorso sono stati i veri e propri protagonisti: sperimentando e operando per tentativi, i più pic- 
coli hanno saputo affrontare molti degli aspetti problematici presenti nel percorso. Quando queste esplorazioni e le relative scoperte venivano presentate ai compagni più grandi, esse venivano valorizzate dal fatto che gli alunni di quinta fossero stimolati a imparare dai più piccoli, implicandosi in attività volte alla comprensione di quanto veniva loro comunicato. Ad esempio, c'è stata un'interessante discussione su quanti assi di legno servissero per fare la base del carretto e molti allievi di quinta ne consideravano solo quattro (tenendo in considerazione le pareti e non il fondo); i bambini della scuola dell'infanzia, che avevano scoperto grazie alla sperimentazione concreta che ne servivano cinque, hanno potuto spiegare questo aspetto ai ragazzi più grandi.

Questi scambi hanno certamente permesso di valorizzare il lavoro di manipolazione ed esplorazione, facendo capire a tutti (docenti compresi) che, nell'affrontare una situazione complessa, il fatto di possedere conoscenze cognitivamente avanzate è tanto importante quanto la possibilità di sperimentare e operare concretamente. Non solo: proprio quest'ultimo fattore rappresenta un tassello fondamentale nell'acquisizione di conoscenze e abilità utili alla risoluzione della situazione problematica. D'altra parte, lo stesso Piano di studio della scuola dell'obbligo ticinese (DECS, 2015) sottolinea l'importanza, soprattutto nei primi cicli di scolarità, di sperimentare e costruire i propri concetti tramite esperienze significative e il confronto con i compagni. Infatti il processo cognitivo "Esplorare e provare" è stato indicato come un aspetto fondamentale (assieme agli altri processi) per la costruzione di un sapere matematico solido e in continua espansione.

Un altro aspetto centrale per il progetto è stato il lavoro sulle capacità di argomentare e comunicare, anch'esso indicato nel Piano di studio come importante processo cognitivo. In quest'ottica, ogni nuova scoperta era condivisa, spiegata, discussa in gruppo, e per ogni problematica erano necessarie nuove ipotesi risolutive da poter verificare. Questo ha in un certo senso obbligato gli allievi a cercare strade comunicativamente efficaci e a trovare termini e strategie per far capire ai compagni cosa avevano scoperto. Vista la grande multietnicità della sezione, si è lavorato molto anche sul vocabolario e sul linguaggio, ponendo l'attenzione sia sui termini più matematici, sia su vocaboli di uso comune.

La situazione - problema ha creato una cornice motivazionale molto forte, grazie alla quale si è mantenuto alto l'interesse e l'impegno degli allievi per tutta la durata del progetto; inoltre, la possibilità di essere i principali protagonisti del percorso, dovendo cercare soluzioni ai problemi concreti riscontrati, ha portato gli allievi a mettersi in gioco, costruire le proprie conoscenze e affrontare le difficoltà con creatività, positività e determinazione.

\section{Il laboratorio come ambiente di lavoro privilegiato}

La modalità di lavoro laboratoriale, già conosciuta dai bambini della sezione di scuola dell'infanzia, è molto ricca e permette un grande coinvolgimento degli allievi (Baldacci, 2004). Sono gli allievi, al loro ritmo e al loro livello, che guidano il progetto, che mettono l'accento su ciò di cui hanno bisogno, che evolvono e fanno evolvere, che scoprono e imparano. Questa metodologia si basa sull'idea che l'insegnante non sia la fonte del sapere, ma guidi gli allievi a fare da soli delle scoperte, a condividerle 
tra loro, a generalizzarle e formalizzarle tutti insieme. Gli allievi, di qualsiasi età, sono fortemente motivati in questo tipo di attività e riescono ad acquisire delle conoscenze e competenze che altrimenti potrebbero rimanere superficiali o poco comprese (D'Amore \& Marazzani, 2011).

Le attività di tipo laboratoriale che abbiamo impostato hanno previsto uno schema sempre simile: gli allievi sperimentano dapprima liberamente, poi vengono messe in comune scoperte, strategie e tentativi fatti, difficoltà e problemi; successivamente si cercano nuove piste o si ipotizzano possibili soluzioni ai problemi riscontrati; infine si torna a sperimentare, creando in questo modo un processo ciclico di costruzione del sapere.

Quando si propone questa modalità di lavoro è interessante vedere come gli allievi riescano a trovare un loro spazio e un loro ritmo. In particolare nella scuola dell'infanzia, dove le differenze d'età tra i bambini portano necessariamente a dover differenziare il lavoro, è interessante notare come in un contesto di tipo laboratoriale la differenziazione avvenga in modo spontaneo e rispettando i ritmi personali: alcuni allievi continuano a sperimentare senza tenere in grande considerazione ciò che è stato proposto dai compagni o dal gruppo, altri hanno bisogno di più tempo e sperimentazione per potersi appropriare delle scoperte fatte e altri ancora sono propositivi e aggiungono e integrano ogni volta qualcosa di nuovo.

Il delicato compito del docente è quello di guidare il percorso come un regista, senza sostituirsi agli allievi, mettendo l'accento sulle problematiche da affrontare, coinvolgendo tutti nel cercare soluzioni, rilanciando soluzioni interessanti già sperimentate, valorizzando l'apporto di allievi che faticano a esporsi nelle discussioni a grande gruppo, e in alcuni momenti aiutando a concentrarsi su ciò che si deve fare per evitare che gli allievi disperdano energie in aspetti non prioritari.

\section{Il progetto}

II progetto si è inserito in un percorso di collaborazione già esistente tra una sezione di scuola dell'infanzia e una classe di quinta elementare. Dopo aver avuto molti momenti di conoscenza e scambio, le due docenti hanno deciso di proporre qualcosa di nuovo e più articolato rispetto alle attività puntuali e slegate tra loro fatte in precedenza.

Si è deciso quindi di lanciare una situazione - problema alla scuola dell'infanzia: la maestra di quinta aveva del materiale da consegnare agli allievi di scuola dell'infanzia ma si trattava di qualcosa di grande e pesante; come avrebbero potuto trasportarlo? Le proposte dei bambini sono state molte; la docente ha cercato di indirizzarli verso una soluzione che potesse essere utile per quel trasporto ma anche in futuro, ampliando quindi la situazione - problema alla seguente: occorre costruire qualcosa per trasportare diverse volte gli oggetti tra la scuola dell'infanzia e la scuola elementare. Le due scuole sono vicine, raggiungibili a piedi tramite stradine pedonali e quindi particolarmente indicate allo scopo.

Dopo aver escluso alcune proposte interessanti ma di difficile realizzazione (per esempio costruire una sorta di teleferica facendo andare un filo dalla scuola dell'infanzia alla scuola elementare), i bambini si sono concentrati sull'idea di costruire un carretto. 
È stato necessario un primo momento per chiarire a tutti che cosa fosse un carretto; essendo una sezione a forte componente multietnica abbiamo fatto in modo di essere certi che ciascun bambino avessero capito di cosa si trattasse. Per questo motivo, inizialmente sono state portate, da bambini e docenti, immagini e descrizioni per illustrare cosa si volesse costruire. Essendo più vicino alla quotidianità dei bambini, il modello di carretto ipotizzato era legato al carrello della spesa (Figura 1), ma grazie alla varietà di esempi proposti (Figura 2) è stato possibile ampliare il bagaglio di rappresentazioni.

Figura 1

Disegno di come un

bambino si rappresentava il carretto.
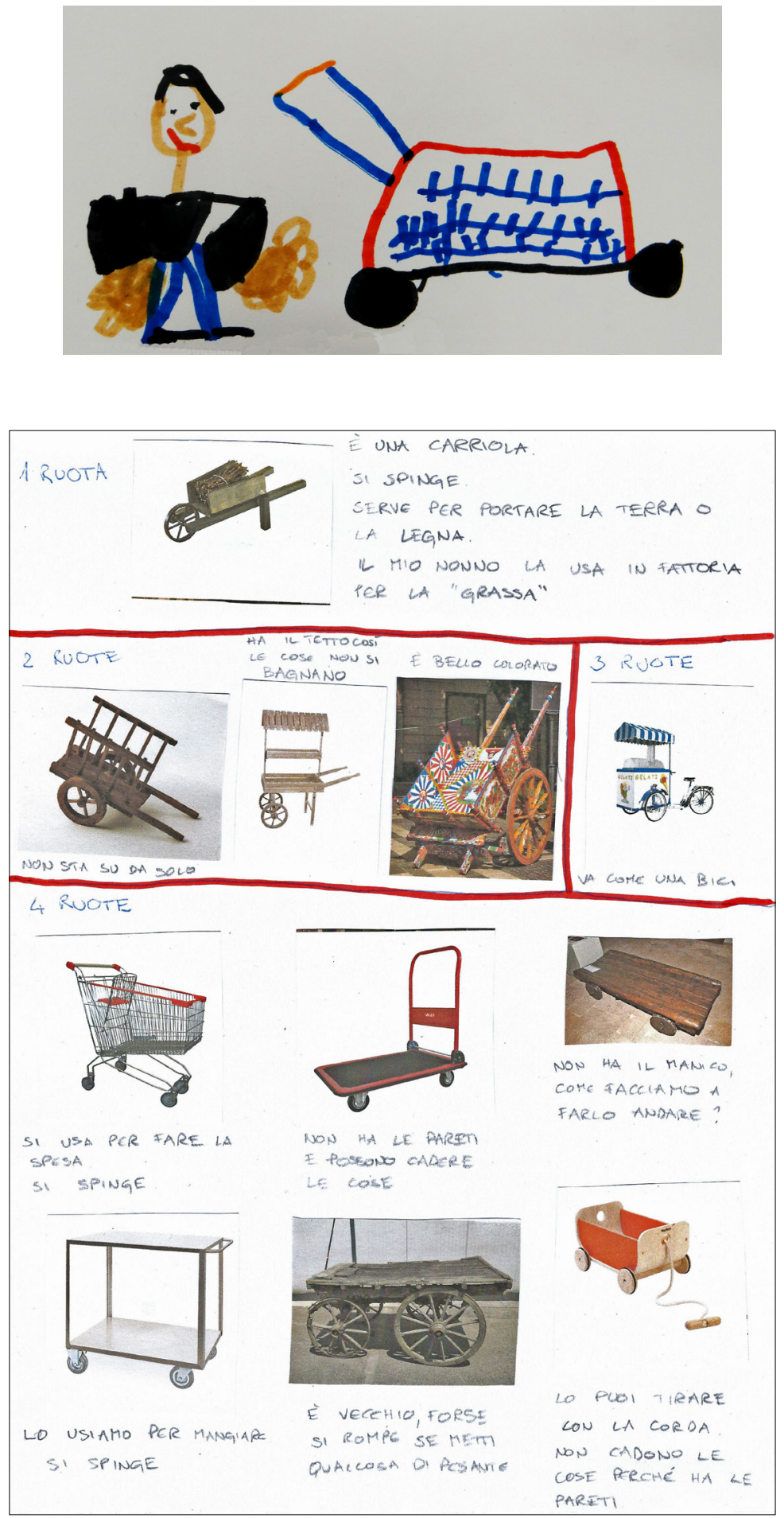
Dopo questa prima necessaria condivisione si è deciso di costruire un carretto con un manico che permettesse di tirarlo. Si è quindi iniziato a discutere su come realizzarlo. Abbiamo proposto ai bambini di iniziare a ideare e costruire dei piccoli modelli realizzati con materiali semplici da gestire, per poi, una volta definito il progetto, farci aiutare dagli allievi di quinta a costruirne uno grande.

Come già affermato, per gli allievi di scuola dell'infanzia il progetto si è basato molto sulla possibilità di sperimentare. Per questo motivo, si è deciso di creare un laboratorio - officina di costruzione di carretti, dove i bambini hanno potuto provare, capire quali erano le difficoltà, cercare singolarmente e tramite messe in comune le soluzioni ai problemi che via via si presentavano e affinare sempre di più il progetto, ampliando così le loro abilità e conoscenze.

Avendo già sperimentato questa modalità didattica, sono stati i bambini stessi a proporre come organizzarsi: hanno allestito un angolo della sezione adibito ad hoc, nel quale hanno messo un tavolo e quattro sedie (perché si poteva entrare solo in quattro alla volta) e hanno deciso di quali materiali e strumenti dotarlo: inizialmente cartoni, fogli, matite, forbici e colle.

A questo punto è iniziata una prima fase di sperimentazione molto libera. I bambini entravano nel laboratorio e provavano a costruire un carretto. Alcuni lo facevano in modo individuale, altri si aiutavano a coppie o in gruppo. Pian piano sono nate esigenze di inserire nuovi materiali: dopo discussioni collettive sono stati così introdotti il nastro adesivo, oggetti rotondi per fare le ruote, bastoncini di vari tipi e lunghezze ecc. A questa prima fase di sperimentazione è seguita una seconda fase, nella quale abbiamo iniziato a mettere in comune i primi risultati (Figura 3).

Figura 3

Alcuni dei primi tentativi di carretto.

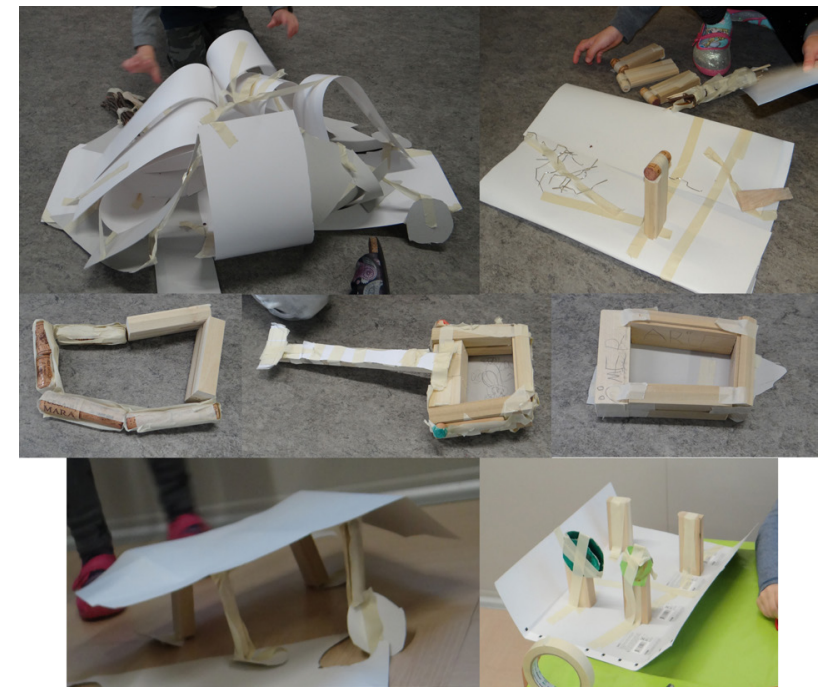

Durante la discussione e il confronto tra i vari modelli creati sono iniziate a emergere importanti considerazioni:

- «Bisogna fare una base di sotto»;

- «Bisogna mettere delle pareti da parte per non far cader fuori le verdure, le cose»;

- «Per fare le pareti ci vogliono dei cartoni uguali, ma se sono diversi si riesce comunque»;

- «Sarà difficile fare le ruote». 
È stato interessante vedere come gradualmente i bambini iniziavano a centrarsi maggiormente sul progetto e, grazie al confronto con i tentativi degli altri, a focalizzare I'attenzione sugli aspetti più rilevanti dando una struttura al risultato che avrebbero voluto ottenere (la base, le pareti, le ruote) e diventando più analitici.

A livello matematico sono iniziate interessanti discussioni, per esempio sulla forma che dovevano avere i cartoni e sulla loro misura, in particolare si è discusso su cosa volesse dire "cartoni uguali" e sul perché fossero necessari trattando, in modo estremamente intuitivo e senza nessun tipo di formalizzazione, il concetto di equiestensione. Con i bambini abbiamo deciso di concentrarci inizialmente sulla parte superiore del carretto e tralasciare l'aspetto delle ruote che sembrava il più complesso da affrontare. I bambini hanno discusso a lungo sulla necessità di trovare un modo per "far star su" le pareti, ed ecco che nel laboratorio hanno sperimentato diverse modalità e soluzioni: con la colla e il nastro adesivo (come illustrato nella Figura 4), tramite I'utilizzo di pieghe o paletti (Figura 5), o arrivando alla fine a capire che se si attaccano le pareti tra loro è più facile che rimangano in piedi (piuttosto che attaccarle solo alla base), come si nota nella Figura 6.
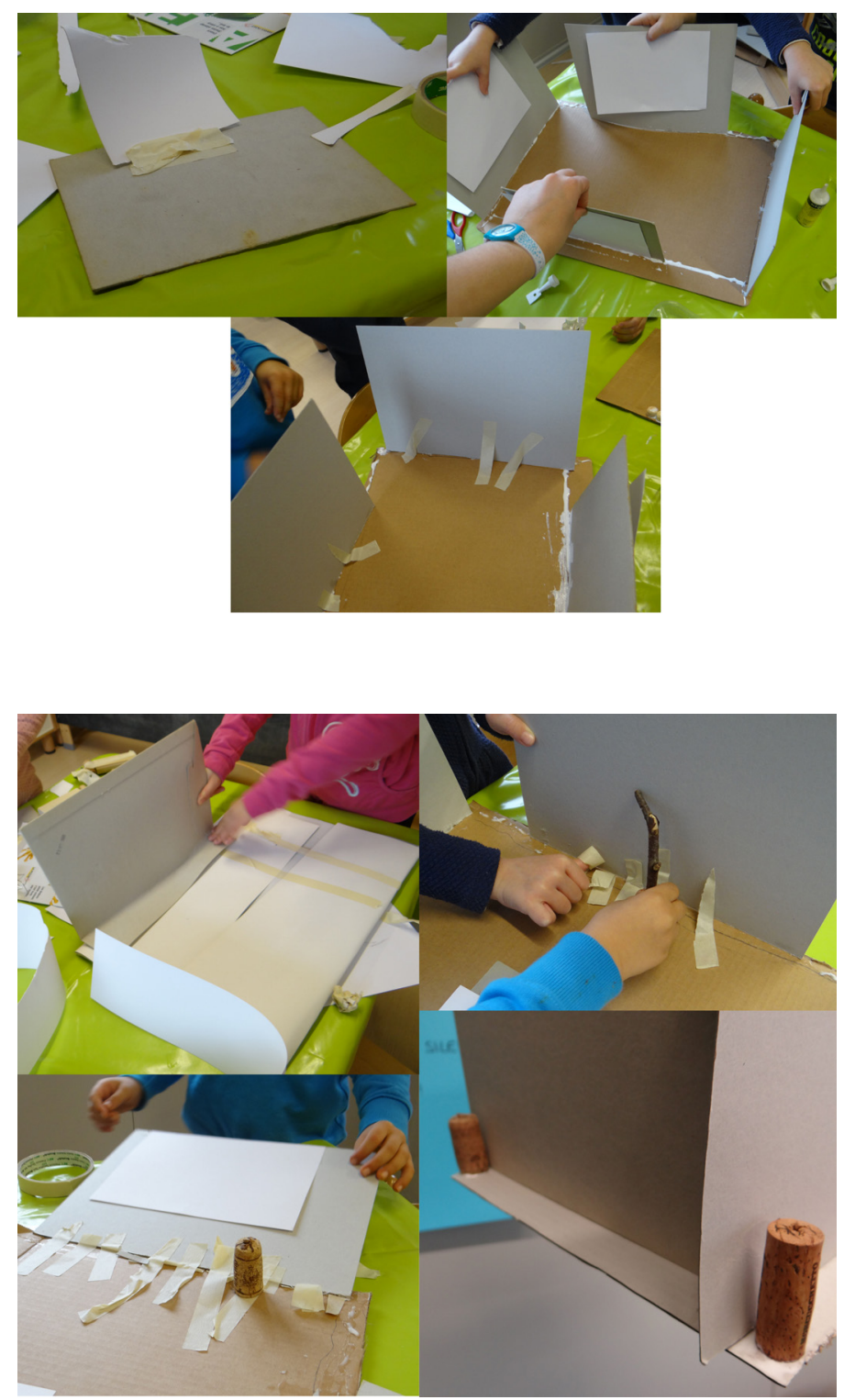
Figura 6

Carretto assemblato

attaccando le pareti tra

loro prima di fissarle alla base.

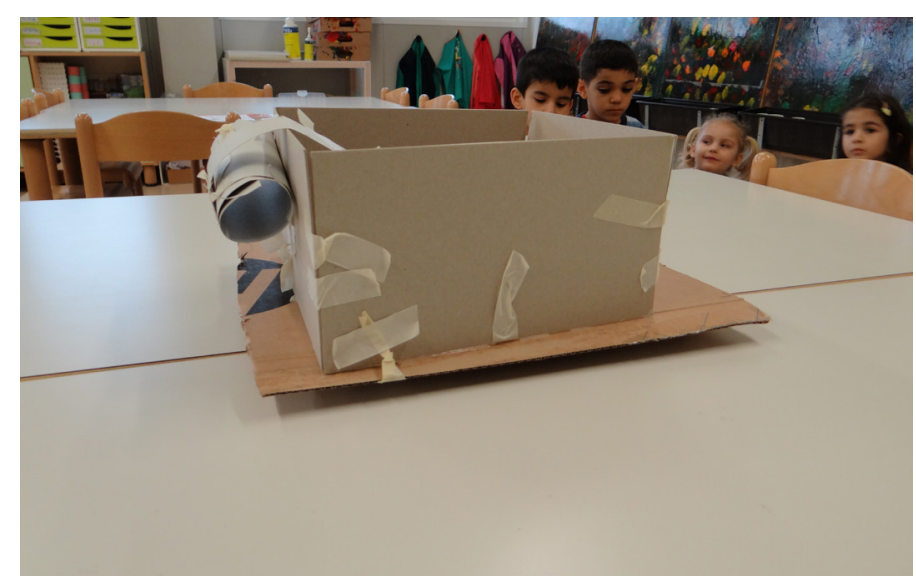

In parallelo abbiamo discusso dei materiali: per realizzare i modelli si stavano utilizzando cartone e carta; che materiale avremmo potuto usare per poi costruire il carretto vero? I bambini hanno subito scartato il cartone perché «se piove si ammollisce» e hanno proposto diverse alternative, spesso lontane da quelle che si aspettava la docente:

- «il cemento perché è forte e se si bagna non si ammollisce»;

- «il ferro perché non si scioglie»;

- «il metallo perché è un po' come il ferro»;

- «il vetro, ma però si può rompere».

Nonostante la validità di alcune di queste proposte spontanee, si è scelto di non far sperimentare ai bambini l'utilizzo di questi materiali per valutarne l'efficacia ma di interpellare gli allievi di quinta, in veste di nostri aiutanti, per chieder loro consiglio. Abbiamo quindi scritto una lettera esponendo le idee avute e chiedendo consiglio. Ci hanno risposto dicendo che delle nostre proposte al limite si poteva tenere in considerazione il ferro ma che loro avrebbero optato per il legno. Dopo averne discusso con i bambini di scuola dell'infanzia, inizialmente scettici perché sostenevano che il legno si sarebbe rotto, e aver osservato quanti mobili e oggetti della scuola dell'infanzia sono costruiti in legno, abbiamo optato per questa soluzione.

È stato dedicato molto tempo alla problematica delle ruote; infatti, man mano che il progetto continuava e le idee si affinavano, emergeva sempre di più l'importanza di una caratteristica delle ruote difficile da realizzare concretamente: la capacità di "girare". I bambini inizialmente attaccavano le ruote utilizzando la colla o il nastro adesivo ma non erano soddisfatti proprio perché in questo modo erano fisse e non giravano.

Abbiamo osservato tutte le ruote che abbiamo trovato in sezione, molti bambini hanno portato oggetti con ruote anche da casa, e abbiamo iniziato a suddividerle in categorie e a capire come fanno a girare. Abbiamo così introdotto nuovi materiali da sperimentare nel laboratorio: tondelli, legnetti, chiodi e viti (e di conseguenza martelli e cacciaviti).

I bambini hanno quindi continuato a sperimentare da questo punto di vista e hanno esplorato le tecniche più efficaci per attaccare le ruote in modo che potessero girare. Alla fine, sempre grazie all'alternanza di sperimentazioni e messe in comune, sono state individuate due modalità, entrambe funzionanti (come si può vedere nella Figura 7):

- «attaccare 2 ruote con il chiodo nel legno da un lato e 2 dall'altro»; 
- «attaccare 2 ruote con un bastoncino lungo, una di qua e una di là, e mettere un bastoncino davanti e uno dietro (ma si deve fare attenzione a come si attacca il bastoncino alla base)».

\section{Figura 7}

Le due modalità trovate per attaccare le ruote.

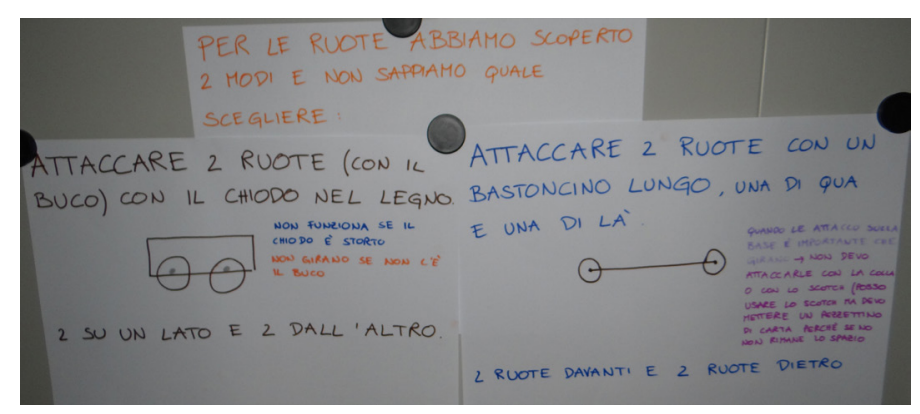

Nel frattempo ci siamo dedicati anche a cercare di formalizzare cosa ci sarebbe servito per costruire il carretto, facendo una sorta di lista della spesa con quantità e descrizioni di tutto il necessario (alcuni esempi nella Figura 8).

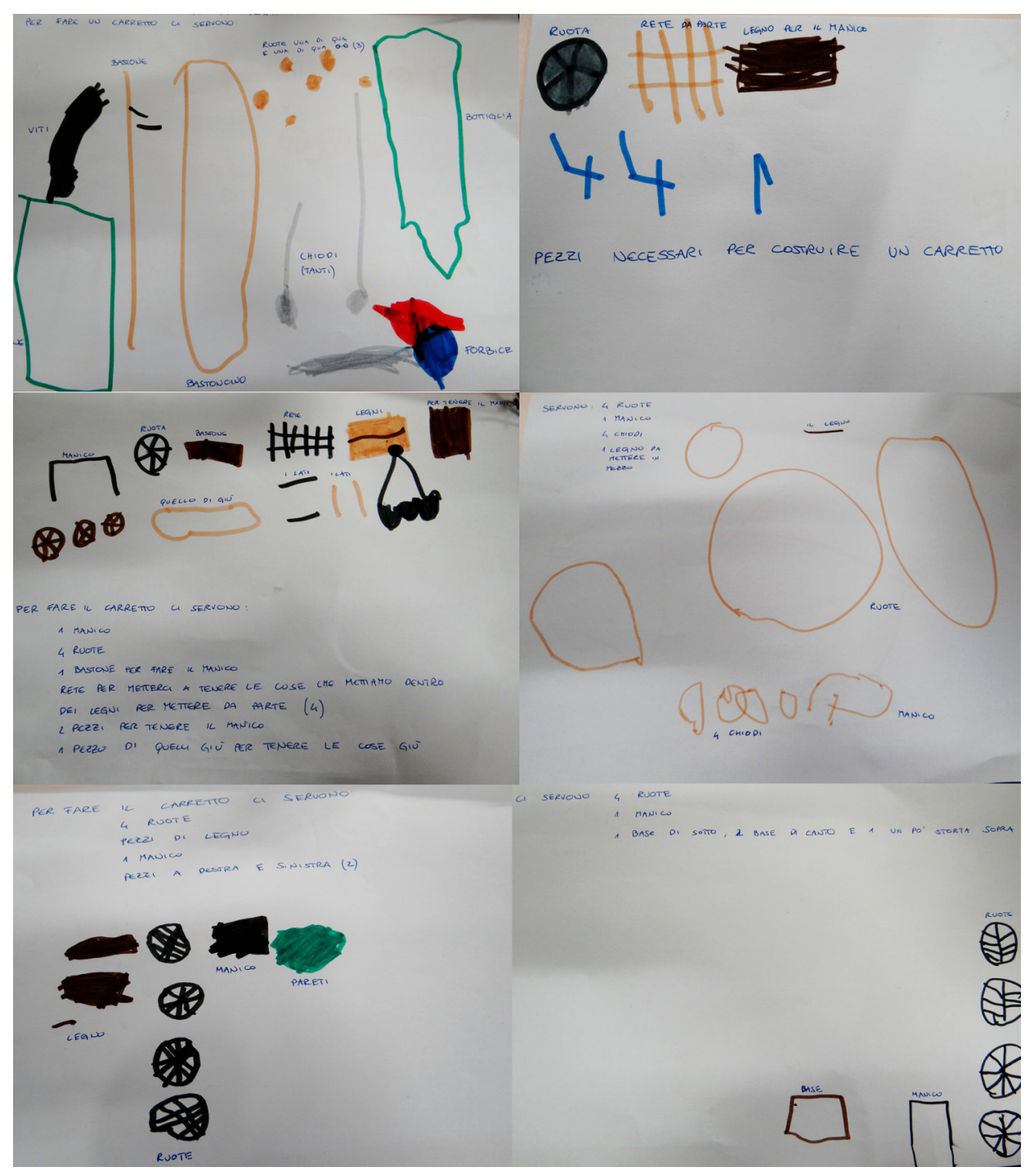


Questo progetto ha coinvolto molto tutti i bambini della sezione e ha permesso una grande differenziazione. Se alcuni bambini più piccoli si sono mostrati attratti solo dalla parte sperimentativa altri erano interessati a più aspetti. Le rappresentazioni del materiale necessario per la costruzione del carretto (Figura 8) sono state volutamente scelte tra prodotti di bambini di livelli diversi, ognuno con le proprie capacità e conoscenze (sia nozionistiche, sia di rappresentazione grafica).

Dal punto di vista matematico, formalizzare il materiale necessario in una lista ha permesso di approfondire molti aspetti, per esempio il nome delle forme geometriche da utilizzare. Abbiamo discusso su come dovessero essere i vari pezzi e quindi anche sulla loro forma, se sul cerchio delle ruote non ci sono state difficoltà abbiamo invece avuto dei problemi a sapere come si chiamasse la forma geometrica necessaria per gli assi di legno, i bambini non conoscevano il termine "rettangolo" (Figura 9).

Figura 9

I bambini di scuola dell'infanzia si interrogano sul nome del rettangolo.

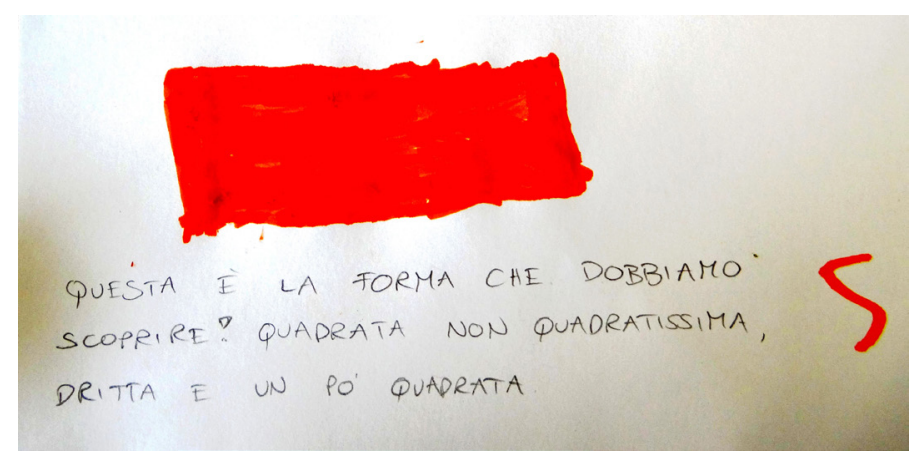

Si è deciso quindi di sfruttare le conoscenze degli allievi di quinta e di interpellarli a questo proposito. Con i bambini di scuola dell'infanzia è quindi stata scritta una lettera:

\footnotetext{
Cari amici della scuola elementare,

volevamo chiedervi come si chiama la forma non rotonda, dritta ma non quadrata, più lunga del quadrato perché noi non lo sappiamo.

Ci serve per sapere la forma della legna per il carretto.

Grazie e speriamo che voi avete delle idee di come si chiama, noi per intanto la chiamiamo asse dritta.

Grazie e a presto
}

I bambini della scuola dell'infanzia Bozzoreda della maestra Laura

I ragazzi di quinta hanno naturalmente avuto molta facilità nel rispondere, si è però deciso di rendere più interessante il loro lavoro chiedendo che spiegassero anche perché si chiama rettangolo e che lo facessero tenendo conto delle conoscenze limitate degli allievi di scuola dell'infanzia che non sanno, per esempio, cos'è un angolo retto o cosa sono due rette parallele.

Ne è quindi nato un interessante lavoro a gruppi nel quale hanno cercato di chiarire e esplicitare aspetti geometrici a loro già conosciuti. Nella Figura 10 si possono vedere alcune delle risposte fornite.

Nel disegnare e rappresentare le figure geometriche si è potuto lavorare molto sulla simbologia, sull'uso intuitivo di numeri o lettere, sulla necessità di affinare il disegno 
e di essere precisi. Nella rappresentazione dei rettangoli necessari per la costruzione del carretto, alcuni bambini hanno spontaneamente iniziato a disegnare, in modo intuitivo, lo sviluppo del solido che rappresentava il carretto come si può vedere nella Figura 11.
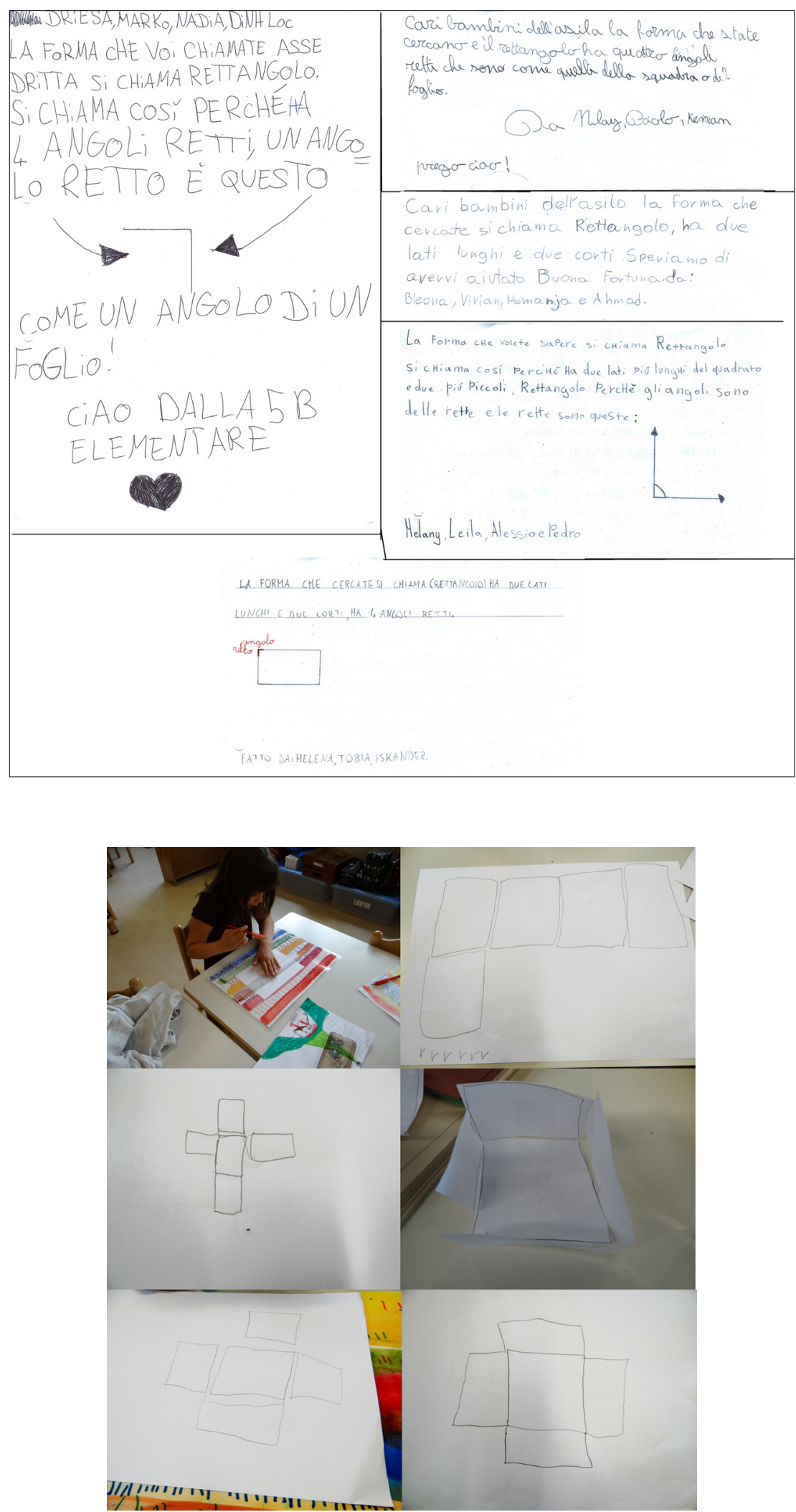
Tornando alla progettazione del carretto, I'ultimo aspetto che abbiamo trattato con i bambini dell'infanzia per avere tutte le informazioni necessarie da dare agli allievi di quinta era quello della dimensione: quanto avrebbe dovuto essere grande il nostro carretto?

Si è trattato di una discussione complessa perché i bambini non avevano nozioni di grandezze e misure, quindi abbiamo cercato di renderla il più concreta possibile scegliendo come supporto l'uso di fogli che rappresentavano l'unità di misura della superficie della base del carretto. Abbiamo fatto vedere agli allievi fogli di varie dimensioni (dall'A5 fino all'A1) e loro hanno scelto la dimensione più congeniale considerando che volevano poter trasportare oggetti grandi. Hanno quindi deciso di avere un carretto con una base grande circa come un A1 $(80 \mathrm{~cm}$ per $60 \mathrm{~cm}$ circa). Per farlo hanno dovuto tener conto del fatto che doveva essere grande per poter contenere tante cose ma anche non troppo grande, perché doveva passare nelle stradine o dalla porta. Per essere certi che non fosse troppo grande abbiamo fatto una prova con un foglio percorrendo tutto il tragitto fino alla scuola elementare. Dal punto di vista matematico, i bambini hanno quindi potuto sperimentare un primo approccio intuitivo al concetto di area e al confronto tra superfici.

Trovate alcune immagini e alcune rappresentazioni dei bambini nella Figura 12.

Figura 12

I bambini di scuola dell'infanzia verificano se la misura scelta per il carretto è adatta per passare nelle stradine.

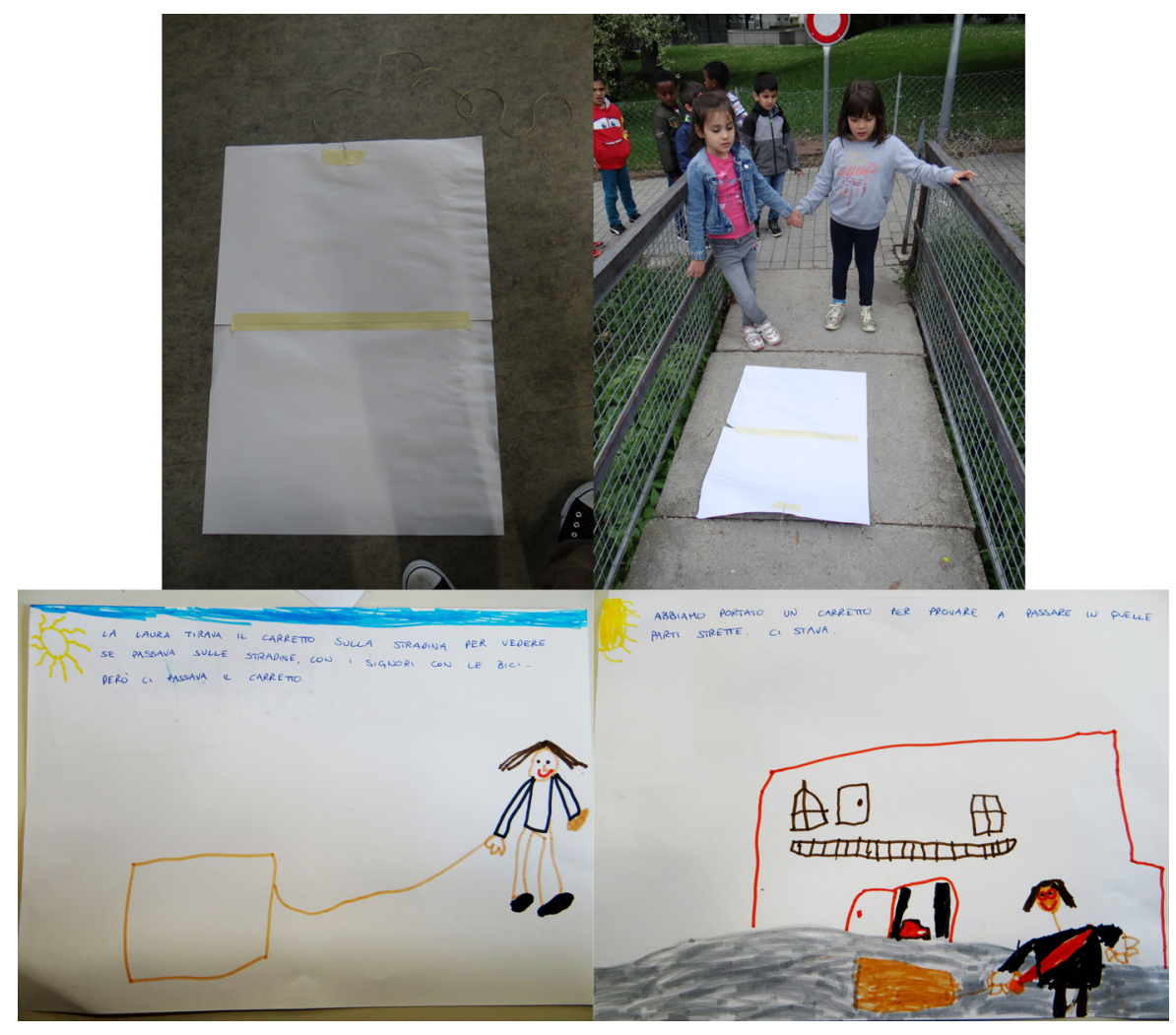

Una volta raccolte tutte le informazioni necessarie, ci siamo recati alla scuola elementare e abbiamo spiegato agli allievi di quinta ciò di cui necessitavamo, chiedendo loro di realizzare un progetto con le misure e il materiale necessario (Figura 13). Abbiamo portato i cartelloni che avevamo via via affinato con tutte le caratteristiche necessarie per il carretto e li abbiamo lasciati a scuola per dare agli allievi di quinta il tempo per elaborare dei progetti. 
Figura 13

I bambini di scuola dell'infanzia spiegano ai compagni di scuola

elementare come deve essere il carretto.

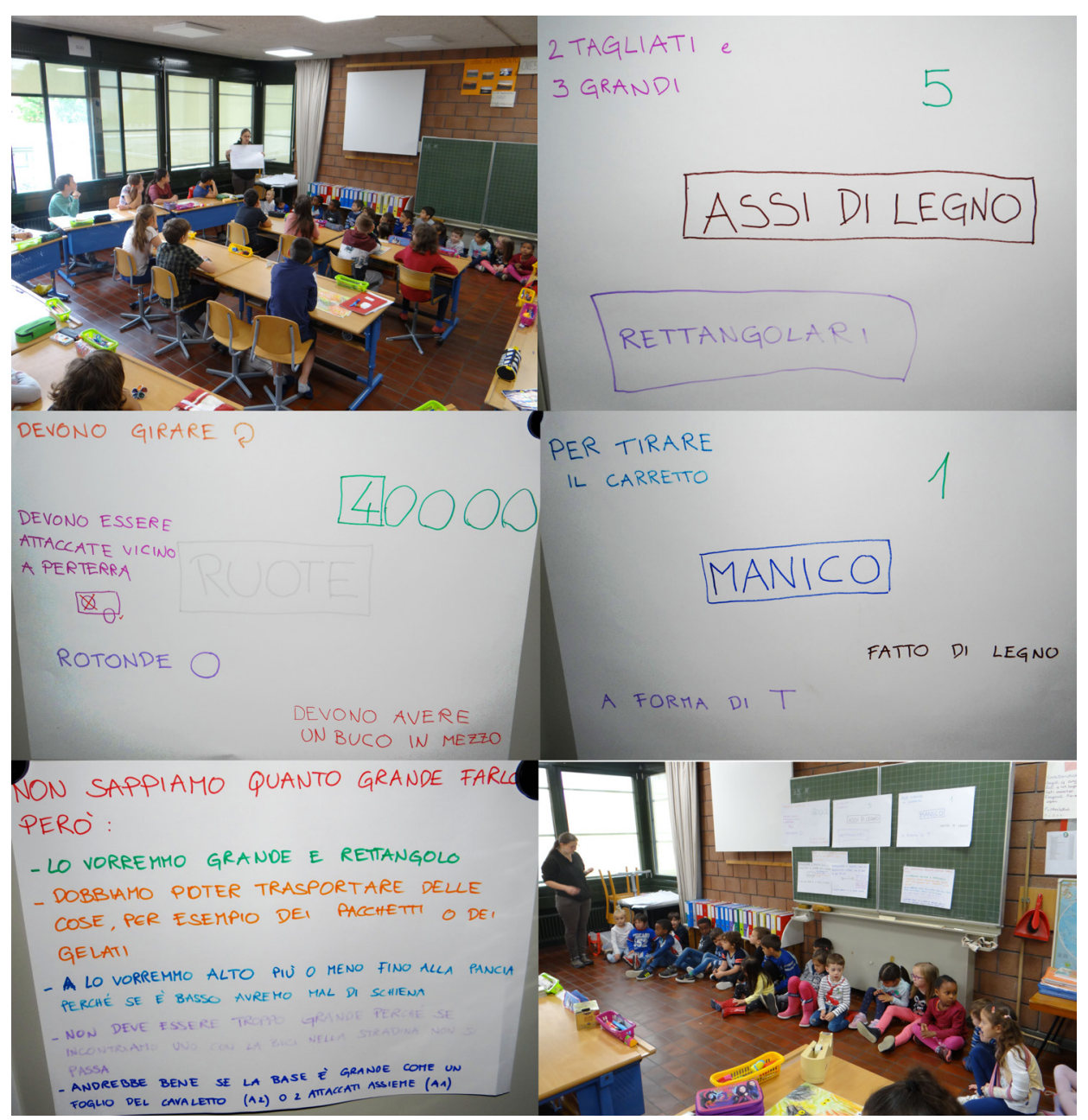

I ragazzi di quinta hanno ricevuto una lista degli assi di legno disponibili in negozio e i prezzi e hanno elaborato dei progetti, dapprima individuali e poi discussi in gruppo. Alcuni ragazzi hanno deciso di sfruttare il disegno in scala, altri hanno fatto degli sviluppi, altri ancora delle istruzioni simili a quelle che si utilizzano per montare i mobili, con una sequenza di ciò che andava fatto. Alcuni esempi sono visibili nella Figura 14.

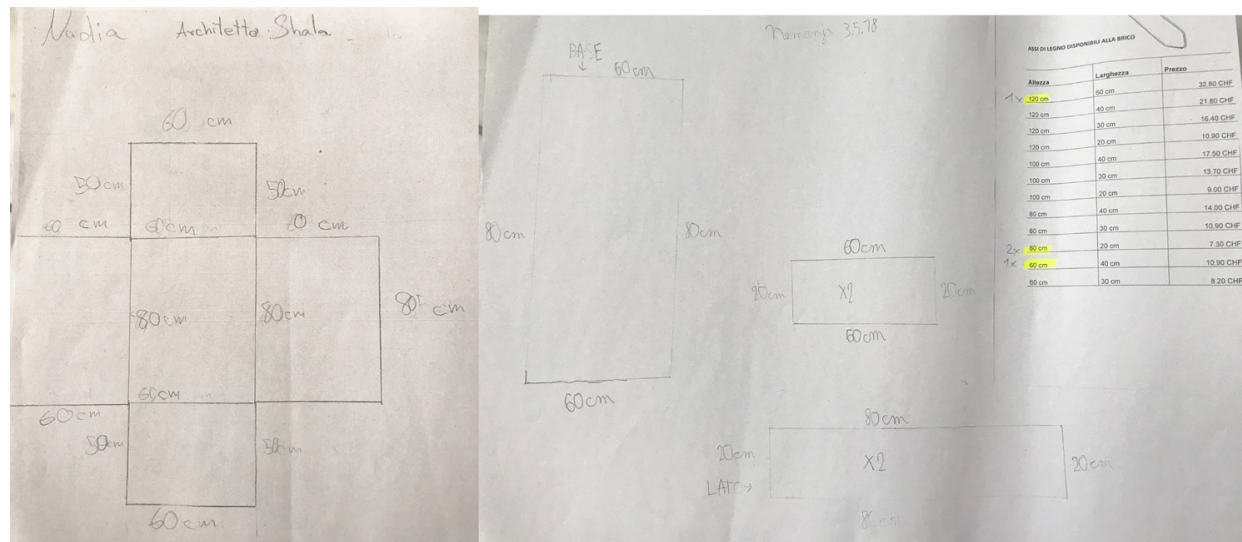


Figura 14

Alcuni progetti elaborat dagli allievi di quinta elementare.

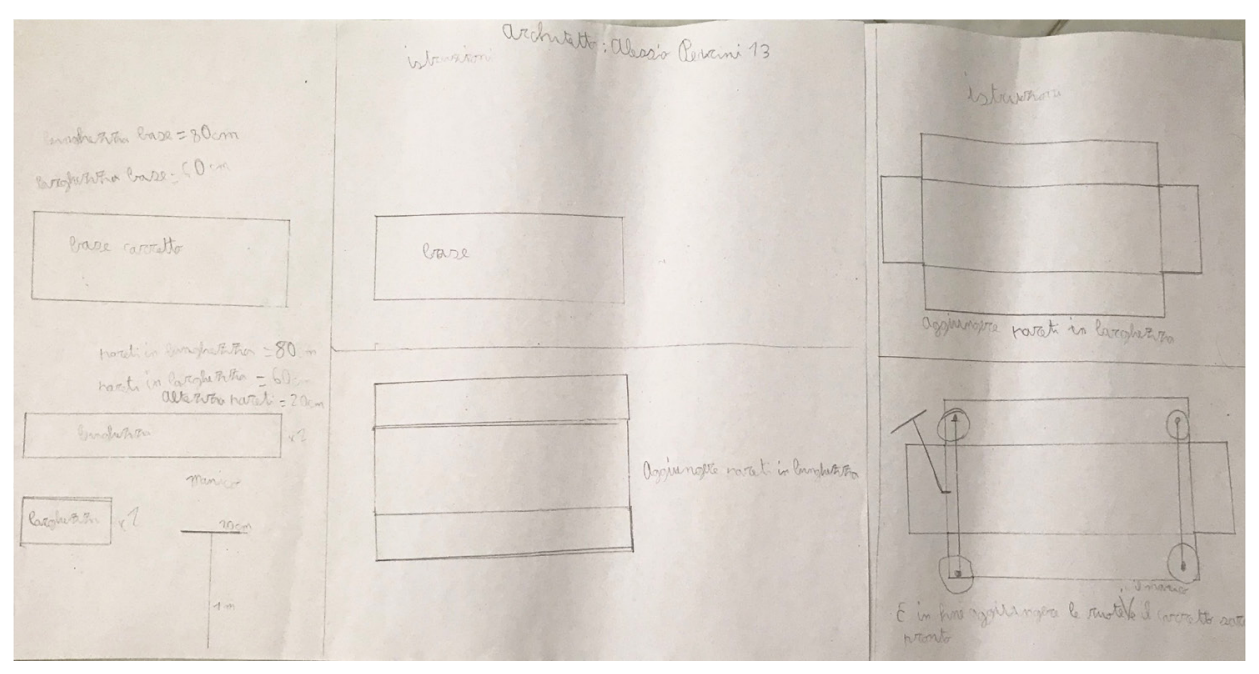

Sono stati molti gli aspetti interessanti per gli allievi di quinta, che si sono trovati confrontati con un compito non semplice; è stato interessante vedere come ognuno abbia saputo spontaneamente sostenere il proprio lavoro con l'aiuto di cui aveva bisogno: alcuni hanno sentito la necessità di costruire un modellino per capire di quanti e quali pezzi avevano bisogno, altri si sono affidati al disegno e altri ancora sono riusciti a farlo già in modo astratto.

A livello matematico hanno potuto sfruttare le conoscenze acquisite sui solidi, sulle aree e sulle misure in generale mettendole in pratica per elaborare un progetto coerente, in modo che le misure degli assi combaciassero tra loro.

I ragazzi di quinta hanno così elaborato dei progetti e ne hanno selezionati alcuni da consegnare ai bambini di scuola dell'infanzia. Si è trattato quindi, per i piccoli, di scegliere quale fosse il più adatto. Per farlo è stata necessaria una prima fase di lettura e comprensione dei progetti e di concretizzazione: una volta compreso che 80 $\mathrm{cm}$ corrispondeva a una misura si trattava di capire quanto fossero lunghi $80 \mathrm{~cm}$ e se corrispondevano alle nostre aspettative. Ė stato quindi svolto un lavoro di analisi che ha portato a conoscere gli strumenti di misura, capire come si potessero utilizzare e rappresentare, grazie a tasselli o scatole, le misure proposte per scegliere quelle più adatte, come si può osservare nella Figura 15.

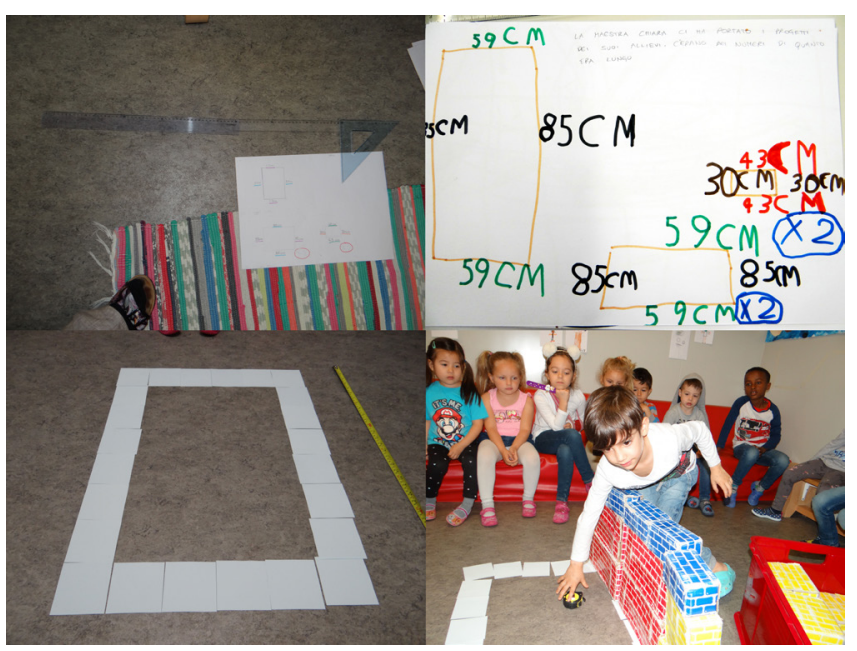


Una volta resi più concreti i progetti, i bambini hanno scelto quello che piaceva loro di più e abbiamo provveduto ad acquistare tutto il materiale necessario.

Purtroppo, per motivi organizzativi (assenza della classe quinta per una settimana di scuola fuori sede) la fase finale di realizzazione concreta del carretto non si è potuta svolgere in collaborazione tra le due classi; abbiamo quindi sfruttato l'aiuto di adulti che hanno dato una mano nella fase di assemblaggio e nelle parti più complesse (utilizzo di trapano, avvitatore ecc.). I bambini di scuola dell'infanzia hanno assistito alla costruzione con grande eccitazione e hanno aiutato laddove potevano (contavano le viti necessarie, mettevano la colla, spiegavano dove si dovevano attaccare i pezzi ecc.). Si possono vedere alcuni di questi passaggi nella Figura 16.

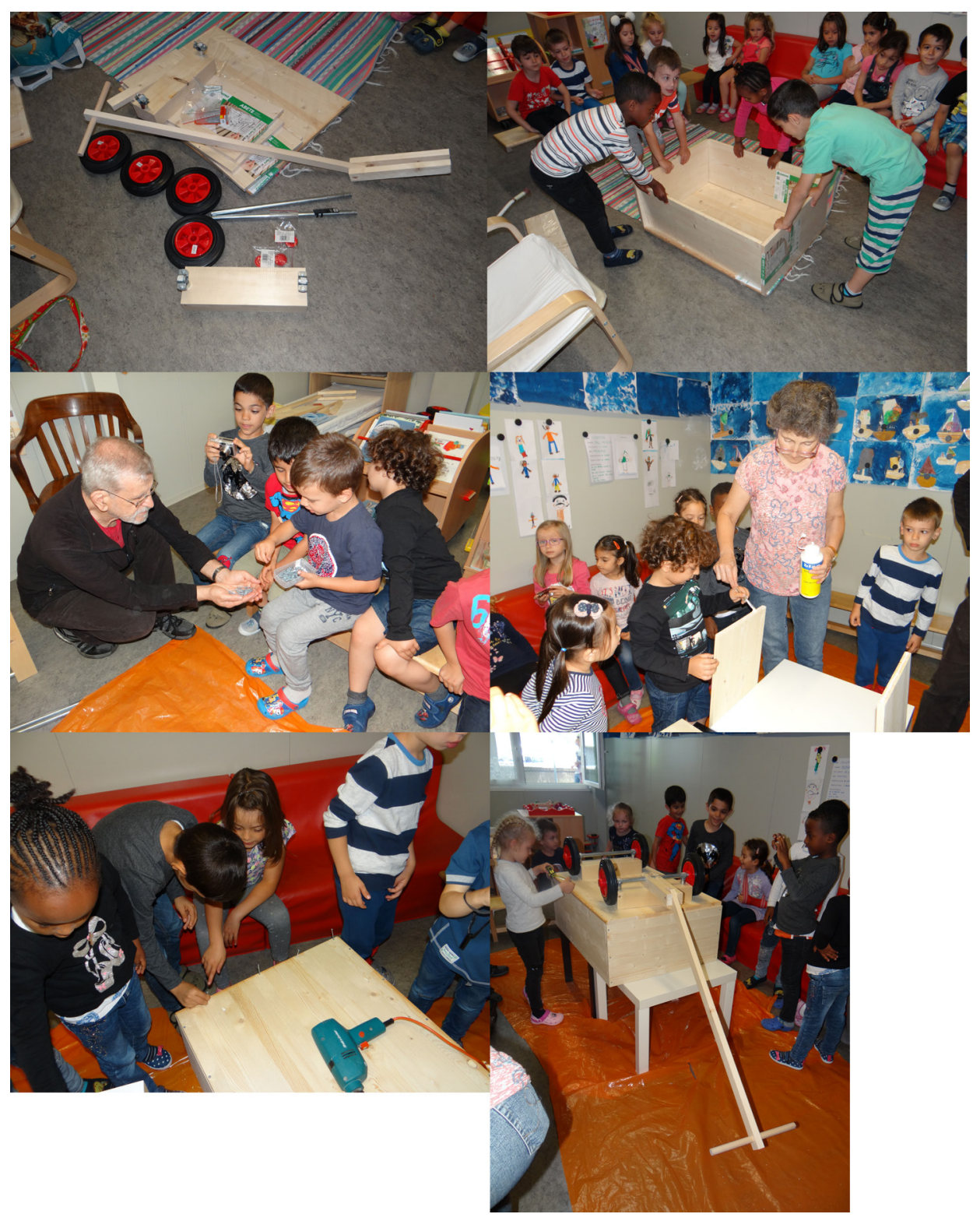

Finalmente, dopo cinque mesi dall'inizio del progetto, abbiamo potuto vedere il frutto del nostro lavoro (Figura 17). 


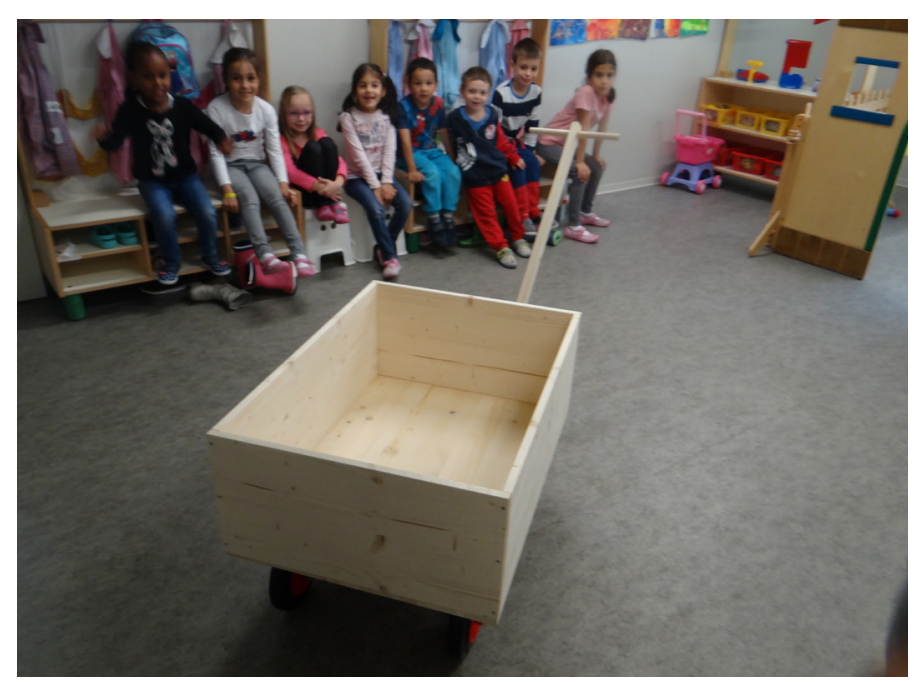

Naturalmente l'abbiamo subito sfruttato per vari trasporti, tra i quali il più importante è stato quello dei gelati per fare merenda assieme ai compagni di quinta elementare. Ora il carretto rimane in sezione e viene utilizzato ogni volta che si presenta l'occasione per creare un collegamento, reale e affettivo, con la scuola elementare.

\section{Conclusioni}

Questo progetto è stato molto ricco e apprezzato dagli allievi e dai docenti. Sono scaturiti molti aspetti interessanti e spunti di lavoro; alcuni sono stati sfruttati, altri potranno essere ripresi in seguito.

Come docenti, abbiamo potuto verificare come la presenza di una situazione problema reale e concreta, vicina al vissuto degli allievi sia una spinta motivazionale molto forte.

Anche la situazione - problema proposta agli allievi di quinta, che non era quella di costruire un carretto, ma di aiutare i bambini di scuola dell'infanzia nel loro percorso, si è rivelata efficace.

Sicuramente questo progetto si sarebbe prestato per poter essere svolto in maniera completa (quindi con la fase di sperimentazione) anche per una classe di scuola elementare. La scelta di far eseguire la sperimentazione solo ai bambini della scuola dell'infanzia è stata presa in base alle condizioni specifiche relative al contesto nel quale operavamo; ciò non toglie che un laboratorio di questo tipo alla scuola elementare sarebbe stato estremamente ricco, offrendo grandi possibilità di crescita per gli allievi. Molti aspetti avrebbero potuto essere approfonditi e maggiormente compresi da tutti gli allievi di quinta se avessero sperimentato concretamente; ad esempio, la necessità di considerare lo spessore del legno nei progetti, oppure il meccanismo di leve relativo al manico del carretto.

La scelta di attribuire agli allievi più grandi il ruolo di "aiutanti" e non di "sperimentatori" ha però permesso di lavorare in modo forte sulla collaborazione e ha creato un fortissimo legame affettivo tra le due classi.

Ora il carretto rimane a disposizione per nuovi progetti di scambio e l'intenzione è 
quella di sfruttarlo per continuare a mantenere forte sia il legame tra i due ordini scolastici sia il messaggio che la collaborazione e la condivisione, non importa con chi, è sempre fonte di arricchimento per tutte le parti (Figura 18).

Figura 18

Il carretto come strumento di unione tra le classi.

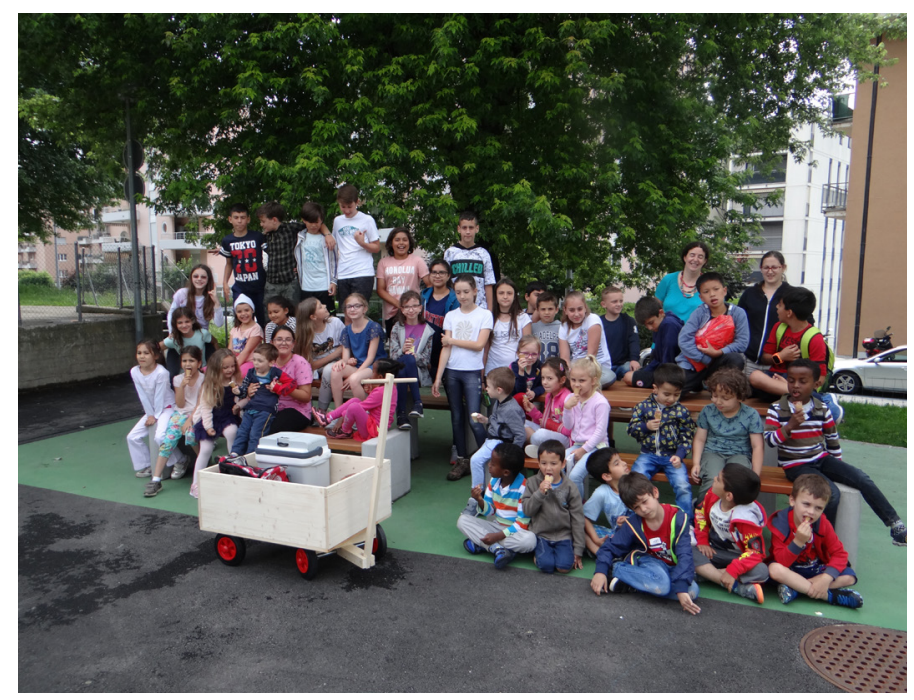

Una descrizione più dettagliata del percorso è disponibile sul sito ScuolaLab tra gli esempi di percorsi didattici in linea con il Piano di studio (https://scuolalab.edu.ti.ch/piazza/ piano di studio). Abbiamo fatto una descrizione sia del percorso degli allievi di scuola dell'infanzia (Mate1i), sia di quello svolto nella classe quinta elementare (Mate2h). Inoltre un breve filmato riassuntivo di tutta l'esperienza è disponibile sullo stesso portale (https://scuolalab.edu.ti.ch/piazza/piano di studio/Matematica/Pagine/Costr uiamo-un-carretto-Mate1i.aspx)

\section{Bibliografia}

Arrigo, G., Maurizi, L., \& Minazzi, T. (2005). "Chi spiega impara a mettere i pensieri bene": la comunicazione intenzionale in matematica. La matematica e la sua didattica, 1, 33-56.

Baldacci, M. (2004). II Laboratorio come strategia didattica. Suggestioni deweyane. In N. Filograsso, R. Travaglini (A cura di), Dewey e l'educazione della mente (pp. 86-97). Milano: Franco Angeli.

Cacciamani, S. (2008). Imparare cooperando: dal Cooperative Learning alle comunità di ricerca. Roma: Carocci.

D'Amore, B., \& Marazzani, I. (2011). Problemi e laboratori. Metodologie per l'apprendimento della matematica. Progetto: Matematica nella scuola primaria, percorsi per apprendere. Bologna: Pitagora.

DECS. (2015). Piano di studio della scuola dell'obbligo ticinese. Bellinzona: Dipartimento dell'educazione, della cultura e dello sport, Divisione della scuola.

\section{Autore/Laura Battaini}

Scuola dell'infanzia - Pregassona, Svizzera

laura.battaini@edu.ti.ch

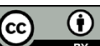

(C) 2019 by the author(s).

DdM 2019 (5), 96 - 112, DOI: $10.33683 / d d m .18 .5 .4$ 